\title{
A study of the morphology and effective density of externally mixed black carbon aerosols in ambient air using a size-resolved single-particle soot photometer (SP2)
}

Yunfei Wu et al.

Correspondence to: Yunfei Wu (wuyf@mail.iap.ac.cn) and Renjian Zhang (zrj@mail.iap.ac.cn)

The copyright of individual parts of the supplement might differ from the CC BY 4.0 License. 
Contents

Fig. S1: Temporal variation in the total number concentration of the scattering and incandescence particles detected by the SP2 during a size selection comprising one short cycle with a duration of $18 \mathrm{~s}$ and one long cycle with a duration of $36 \mathrm{~s}$ for each size.

Fig. S2: Frequency distribution of the lag times between the incandescence and scattering peak locations determined using the SP2.

Fig. S3: The calibration curves for the relationship between the SP2-measured incandescence peak height (low gain) and the refractory $\mathrm{BC}(\mathrm{rBC})$ mass.

Fig. S4: Detection efficiency of the SP2 as a function of (a) the mobility diameter of Aquadag and (b) the mass-equivalent diameter of $\mathrm{rBC}$.

Fig. S5: Frequency distribution of the $d_{\mathrm{me}}$ of $\operatorname{ext} \mathrm{BC}$ at 16 prescribed mobility sizes (in the $140-750 \mathrm{~nm} d_{\mathrm{mob}}$ range) analyzed in the current study.

20 Fig. S6: The same as Fig. S5 but for the normalized number size distribution $\left(\mathrm{d} N / \mathrm{d} \log D_{\mathrm{c}}\right)$.

Fig. S7: Time series of hourly $\mathrm{PM}_{2.5}$ mass concentrations measured at the same site during the experimental period.

Fig. S8: The mass of ext $\mathrm{BC}$ particles as a function of the mobility diameter in the range of 140-750 nm during the polluted episode and the clean period.

Fig. S9: The number fraction of ext $\mathrm{BC}$ as a function of the mobility diameter $\left(d_{\mathrm{mob}}\right)$ during the polluted episode and the clean period.

Table S1: The typical mass-equivalent diameters $\left(d_{\mathrm{me}}\right)$ and corresponding masses of ext $\mathrm{BC}$ at different mobility sizes $\left(d_{\mathrm{mob}}\right)$ selected by the DMA throughout the whole campaign. 


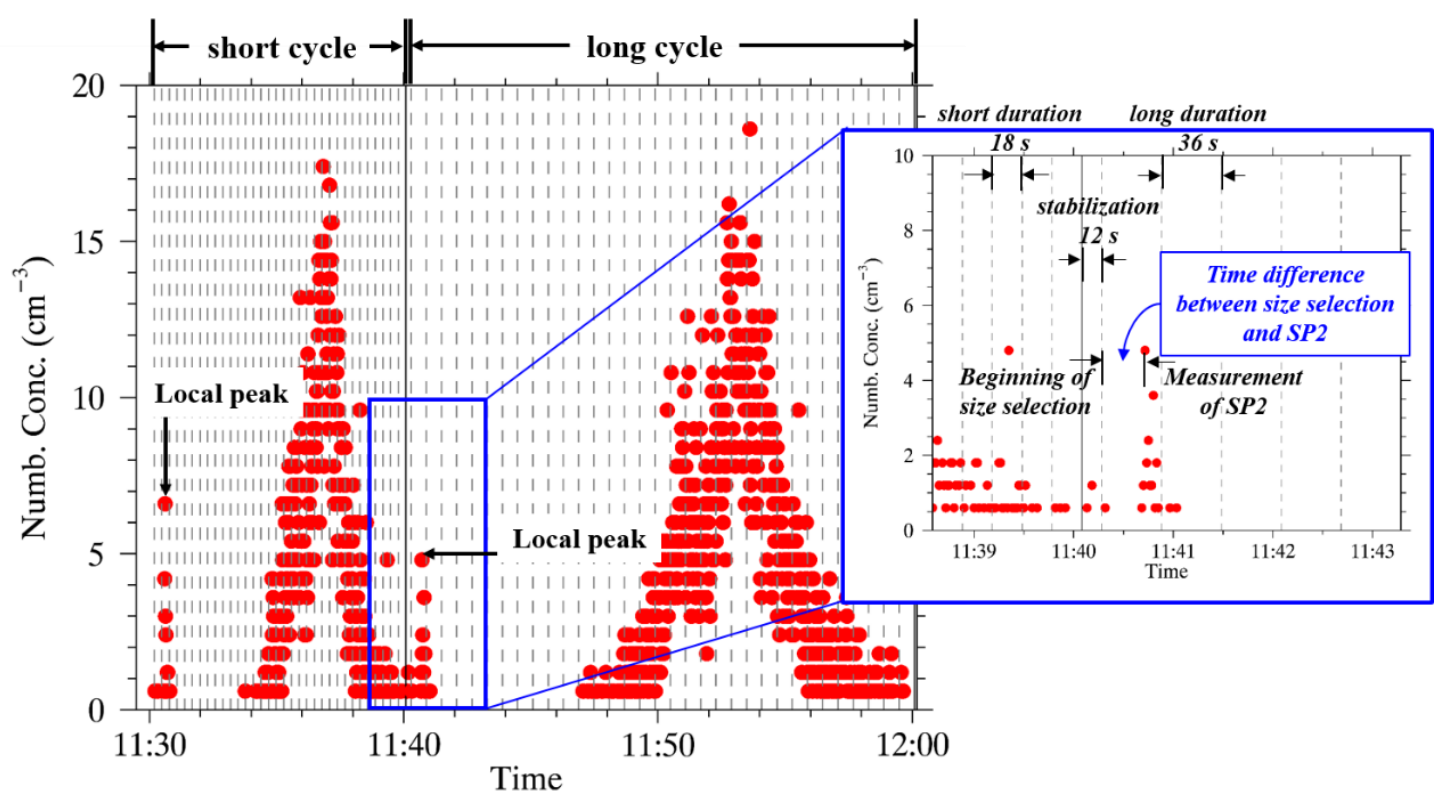

30 Fig. S1: Temporal variation in the total number concentration of the scattering and incandescence particles detected by the SP2 during a size selection comprising one short cycle with a duration of $18 \mathrm{~s}$ and one long cycle with a duration of $36 \mathrm{~s}$ for each size. The local peak at the beginning of each cycle was first identified to correct the time difference between the DMA size selection and the SP2 measurement. 


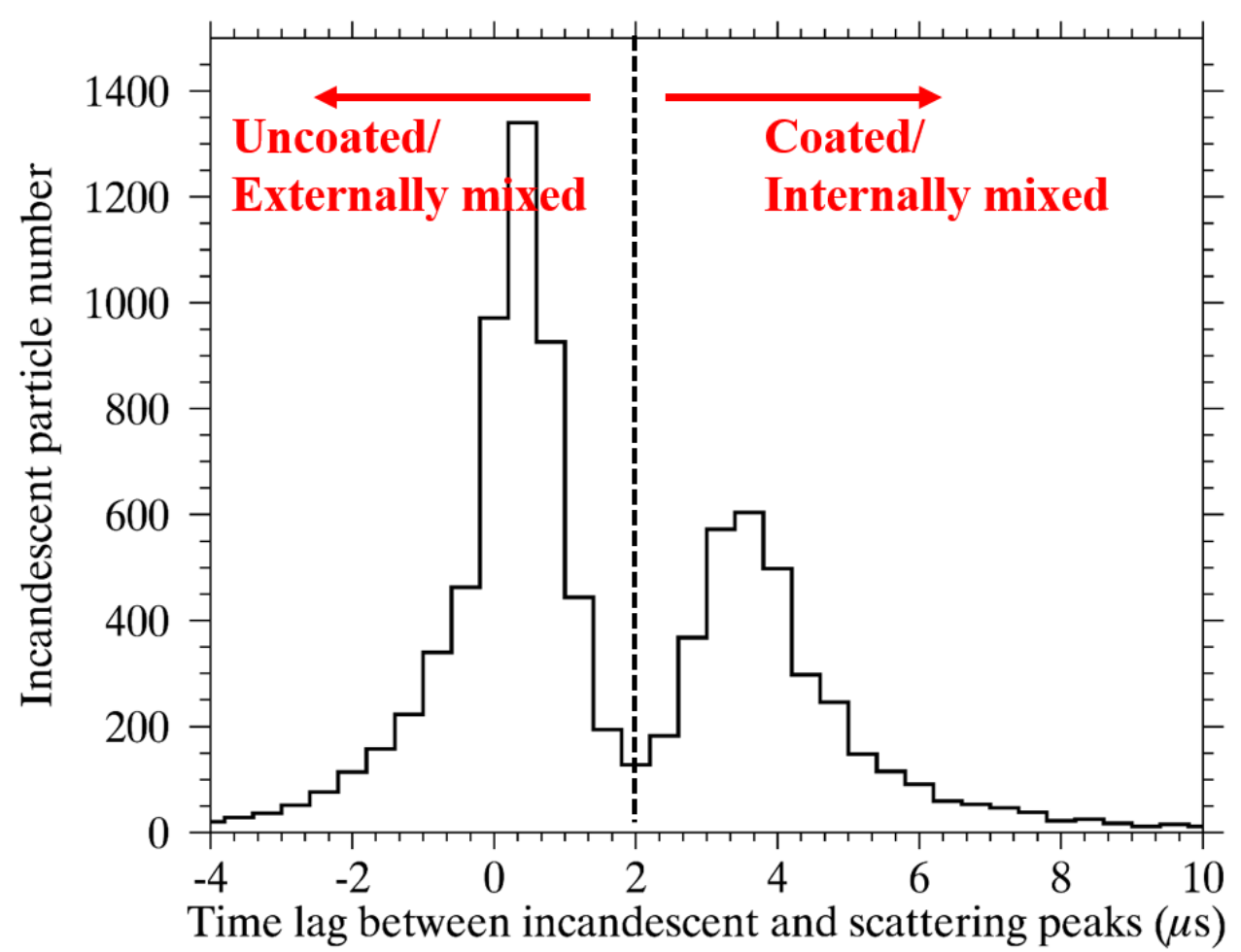

Fig. S2: Frequency distribution of the lag times between the incandescence and scattering peak locations determined using SP2. A bimodal distribution is found, with the minimum at $\sim 2 \mu \mathrm{s}$. The BC-containing particles with a lag time greater than $2 \mu$ s were considered to be thickly coated. Otherwise, the BC-containing particles were uncoated or thinly coated. 


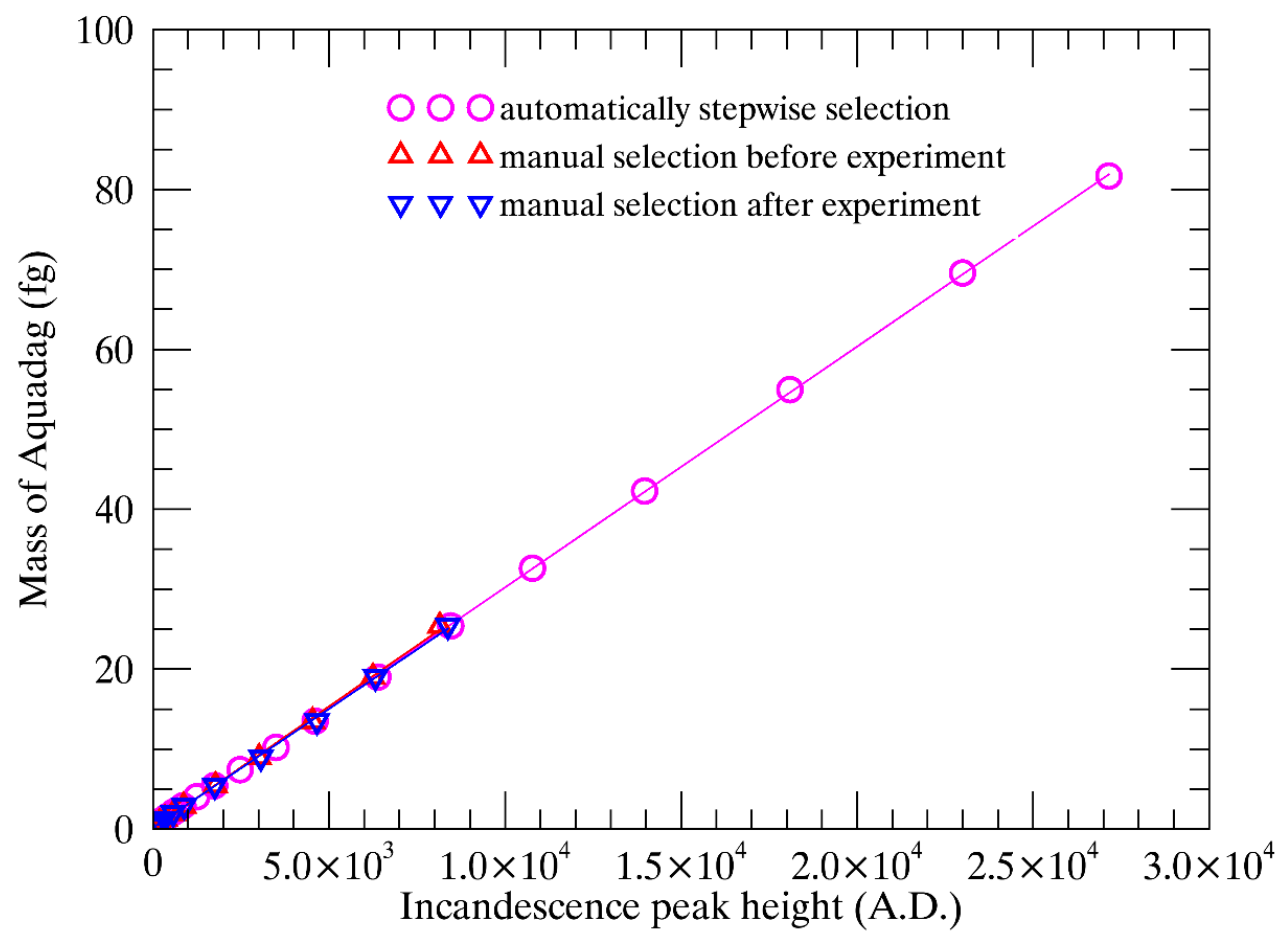

Fig. S3: The calibration curves for the relationship between the SP2-measured incandescence peak height (low gain) and the refractory $\mathrm{BC}(\mathrm{rBC})$ mass. Aquadag particles were used as the representative material of $\mathrm{rBC}$ in the calibration.

45 Aquadag particles with different mobility sizes $\left(d_{\mathrm{mob}}=100,125,150,175,200,250,300,350,400,450 \mathrm{~nm}\right.$, respectively) were manually selected by a DMA. The masses of the size-selected Aquadag particles were calculated based on the effective densities $\left(\rho_{\text {eff }}\right)$ according to the density-mobility relationship provided by Gysel et al. (2011). The manual calibrations were performed both before (on 23 Jan. 2018) and after the campaign (on 10 Feb. 2018). The red upward triangles represent the relationship between the mass of Aquadag and the incandescence peak height during the calibration before the campaign. The blue downward triangles represent the calibration after the campaign. It is clear that the SP2 incandescence peak height is proportional to the mass of the Aquadag particles, yielding the linear regression functions of $y=0.003048 x+0.1058\left(R^{2}=0.9991\right)$ for the calibration before the campaign and $y=0.002972 x+0.1713$ $\left(\mathrm{R}^{2}=0.9992\right)$ for the calibration after the campaign. In the functions, $y$ represents the mass of the Aquadag particles, and $\mathrm{x}$ represents the incandescence peak height. To examine the detection efficiency of the SP2, the size-resolved Aquadag 55 particles were also automatically selected by the DMA in the $d_{\text {mob }}$ range of 140-750 nm and then measured using the SP2 and CPC after the campaign. The mass-incandescence relationship derived from this measurement was very close to the manual calibrations. The linear regression function is $y=0.003012 x+0.1097\left(R^{2}=0.9999\right)$. The similar $r B C$ mass calibration curves indicate that the SP2 operated very stably throughout this campaign. Considering that the incandescence signal peaks of the Aquadag particles were $~ 25 \%$ higher than those of the ambient BC particles, the slopes of the calibration equations should be multiplied by a factor of $\sim 1.33$ when converting the measured incandescence signal peaks to the rBC masses for ambient particles. 

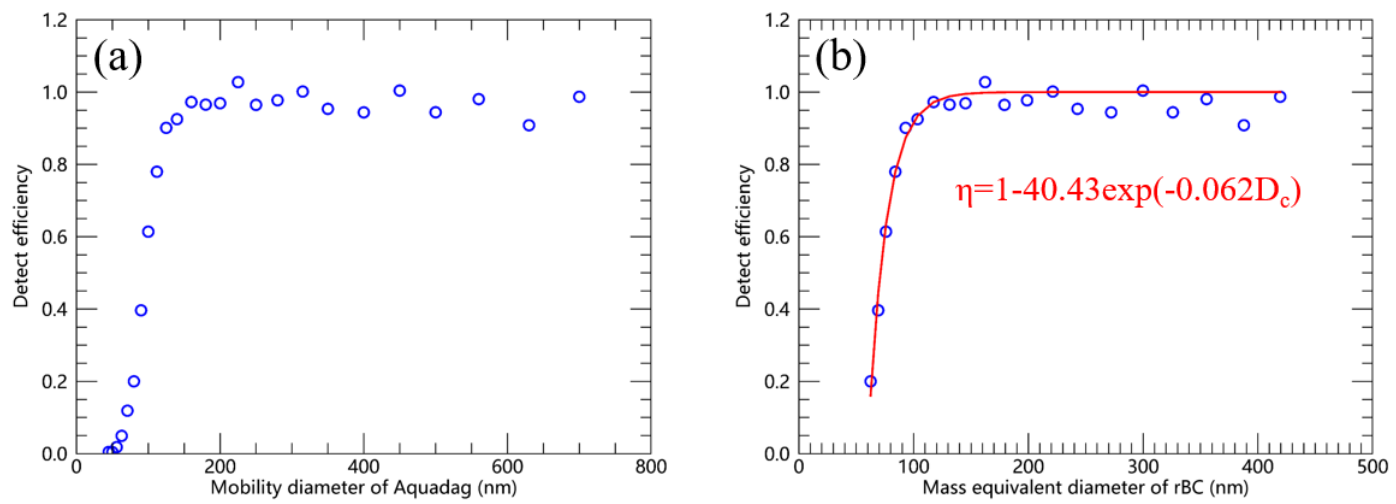

Fig. S4: Detection efficiency of the SP2 as a function of (a) the mobility diameter of Aquadag particles and (b) the massequivalent diameter of $\mathrm{rBC}$. The detection efficiency was calculated as the ratio of the number concentration of particles 65 detected by the SP2 to that measured in parallel by the CPC at prescribed mobility sizes. Since the Aquadag particles were generated by an aerosol generator, pure scattering particles should not exist. The particles with detectable incandescence signals were recorded by the SP2. It is clear that adequate detection efficiencies (> 90\%) for the SP2 were observed when the $d_{\mathrm{mob}}$ of the Aquadag particles was larger than $125 \mathrm{~nm}$. Correspondingly, the mass-equivalent diameter of $\mathrm{rBC}$ calculated using the $d_{\mathrm{mob}}$ and $\rho_{\text {eff }}$ of the Aquadag particles should be larger than $\sim 90 \mathrm{~nm}$ to provide

70 adequate detection efficiencies. The relationship between the detection efficiency of the SP2 and the rBC mass can be characterized using an exponential function, as shown in Fig. S4b. 

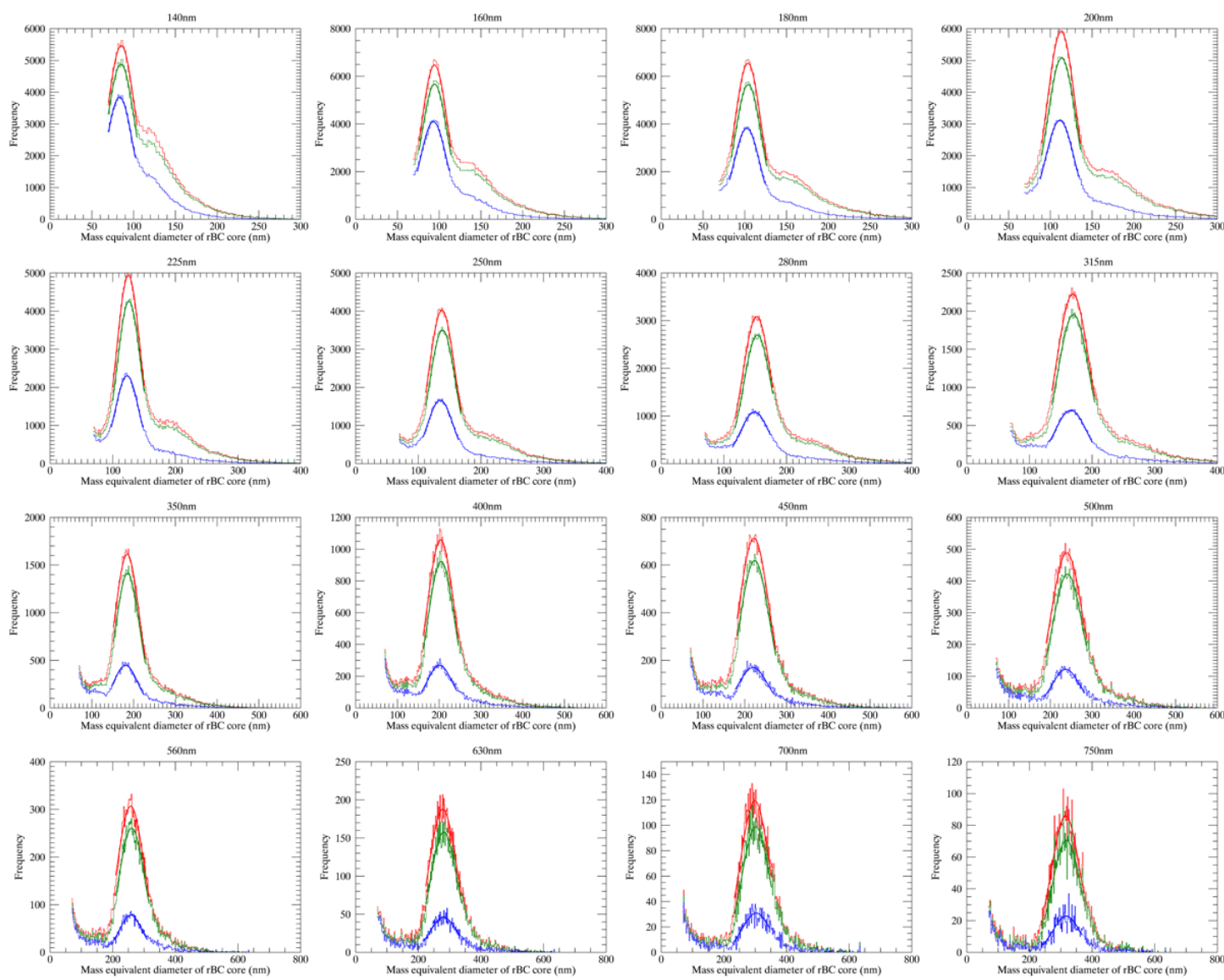

Fig. S5: Frequency distribution of the $d_{\mathrm{me}}$ of $\operatorname{ext} \mathrm{BC}$ at 16 prescribed mobility sizes (in the $140-750 \mathrm{~nm} d_{\text {mob }}$ range) analyzed in the current study. Three thresholds of delay time between the incandescence signal peak and the scattering peak detected by the SP2 were employed to discriminate the extBC particles, e.g., $2.0 \mu \mathrm{s}, 1.2 \mu$ s and $0.4 \mu \mathrm{s}$. The frequency distributions corresponding to the thresholds are shown in red, green and blue colors, respectively. Reducing the delay time threshold results in a decrease in the data volume used in the statistics, while it seems to have few effects on the peak locations of the distributions. The major peak of the frequency distribution at smaller mobility sizes with a $d_{\mathrm{mob}}<140$ $\mathrm{nm}$ is not clear due to the lower limit of the SP2. Thus, these smaller sizes were not discussed in the current study. 

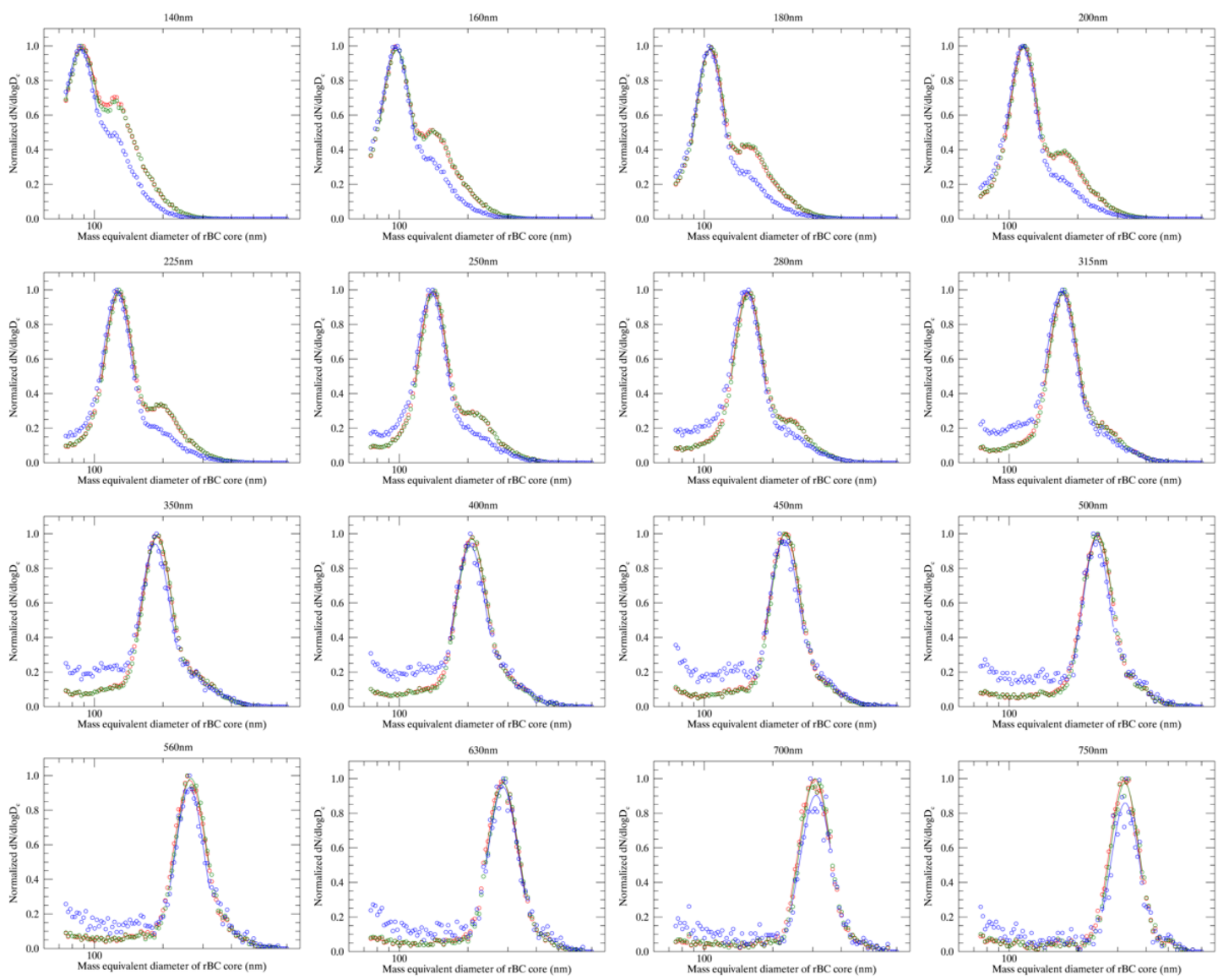

Fig. S6: The same as Fig. S5, but for the normalized number size distribution $\left(\mathrm{d} N / \mathrm{d} \log D_{\mathrm{c}}\right)$. The quantity $D_{\mathrm{c}}$ indicates the mass-equivalent diameter of the $\mathrm{rBC}$ core, the same as $d_{\mathrm{me}}$ defined above. Compared to Fig. S5, these graphs more clearly show that reducing the time-delay threshold has few effects on the peak $d_{\mathrm{me}}$. The main changes occur in the right tail of the distributions at each mobility size. Reducing the time delay threshold results in a significant reduction of particles in the right tail of the number size distribution. These particles are likely to be thinly or even moderately coated $\mathrm{BC}$ particles, which were also recognized as ext $\mathrm{BC}$ using the time delay approach. Because the peak $d_{\mathrm{me}}$ values are needed in the current study and are considered the typical $d_{\text {me }}$ values for the prescribed $d_{\mathrm{mob}}$, these thinly or even moderately coated BC particles should have few effects on our results presented in this study. The peak $d_{\text {me }}$ value is identified as the mode value of a lognormal function that is fitted to the major peak of size distribution at each mobility. 
Polluted episode Clean period

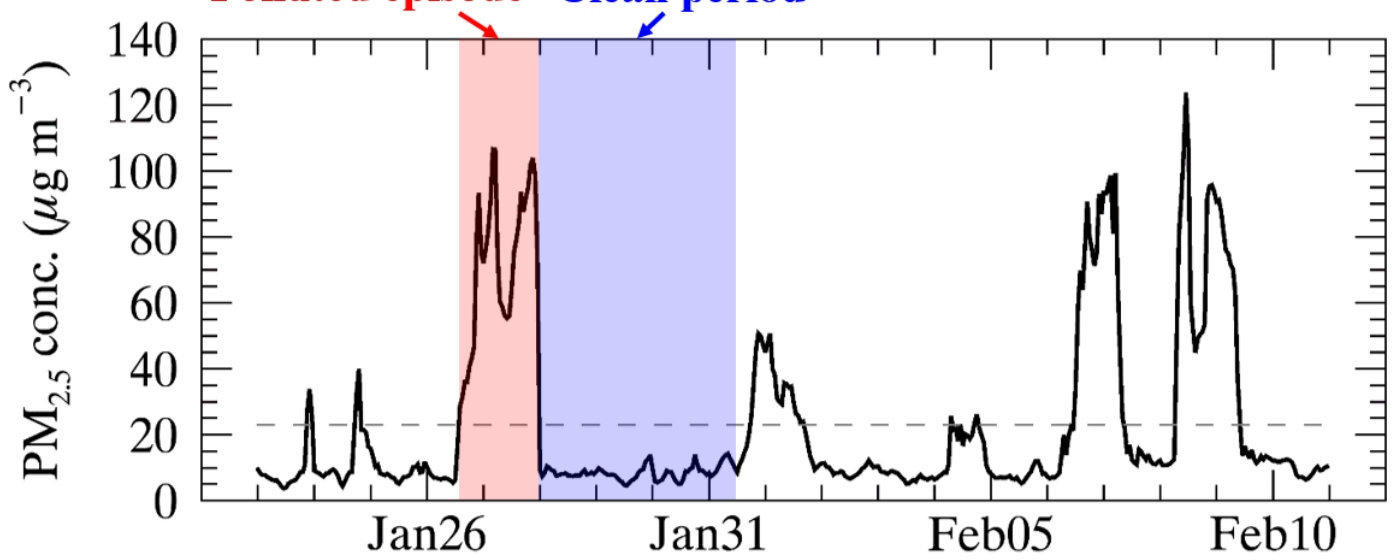

Fig. S7: Time series of hourly $\mathrm{PM}_{2.5}$ mass concentrations measured at the same site during the experimental period. The 95 dashed line represents the mean $\mathrm{PM}_{2.5}$ mass concentration with a value of $23.0 \pm 26.7 \mu \mathrm{g} \mathrm{m}^{-3}$. The red and blue shaded regions represent a polluted episode (mean $\mathrm{PM}_{2.5}=72.1 \pm 23.1 \mu \mathrm{g} \mathrm{m}^{-3}$ ) and a subsequent clean period (mean $\left.\mathrm{PM}_{2.5}=8.9 \pm 2.7 \mu \mathrm{g} \mathrm{m}^{-3}\right)$, respectively, in which the mass-mobility relationships of ext $\mathrm{BC}$ are compared. 


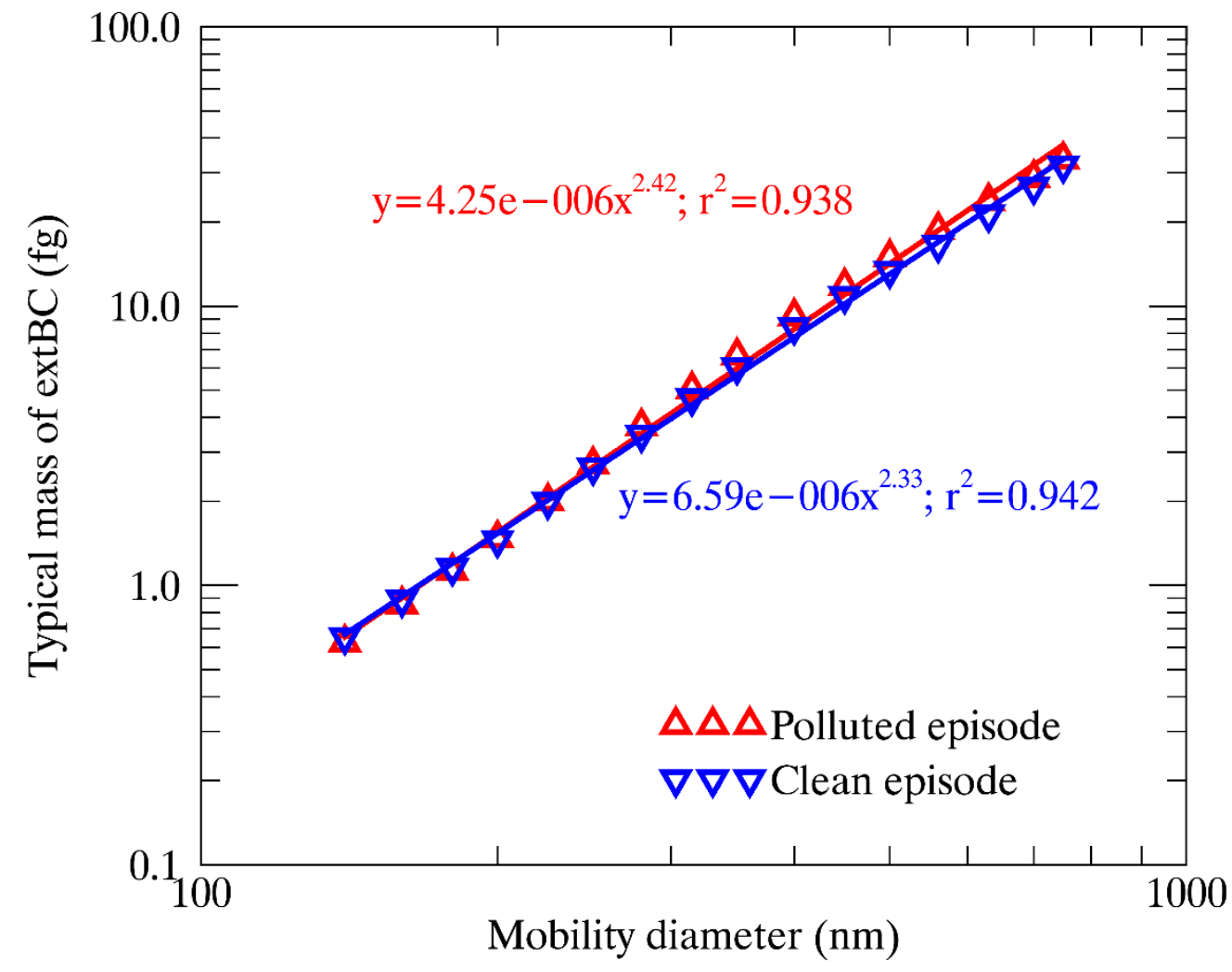

100 Fig. S8: The mass of extBC particles as a function of the mobility diameter in the range of 140-750 nm (black circles) in the polluted (red upward triangles) and clean (blue downward triangles) episodes. The power-law functions are fitted to the mass-mobility relationships. 


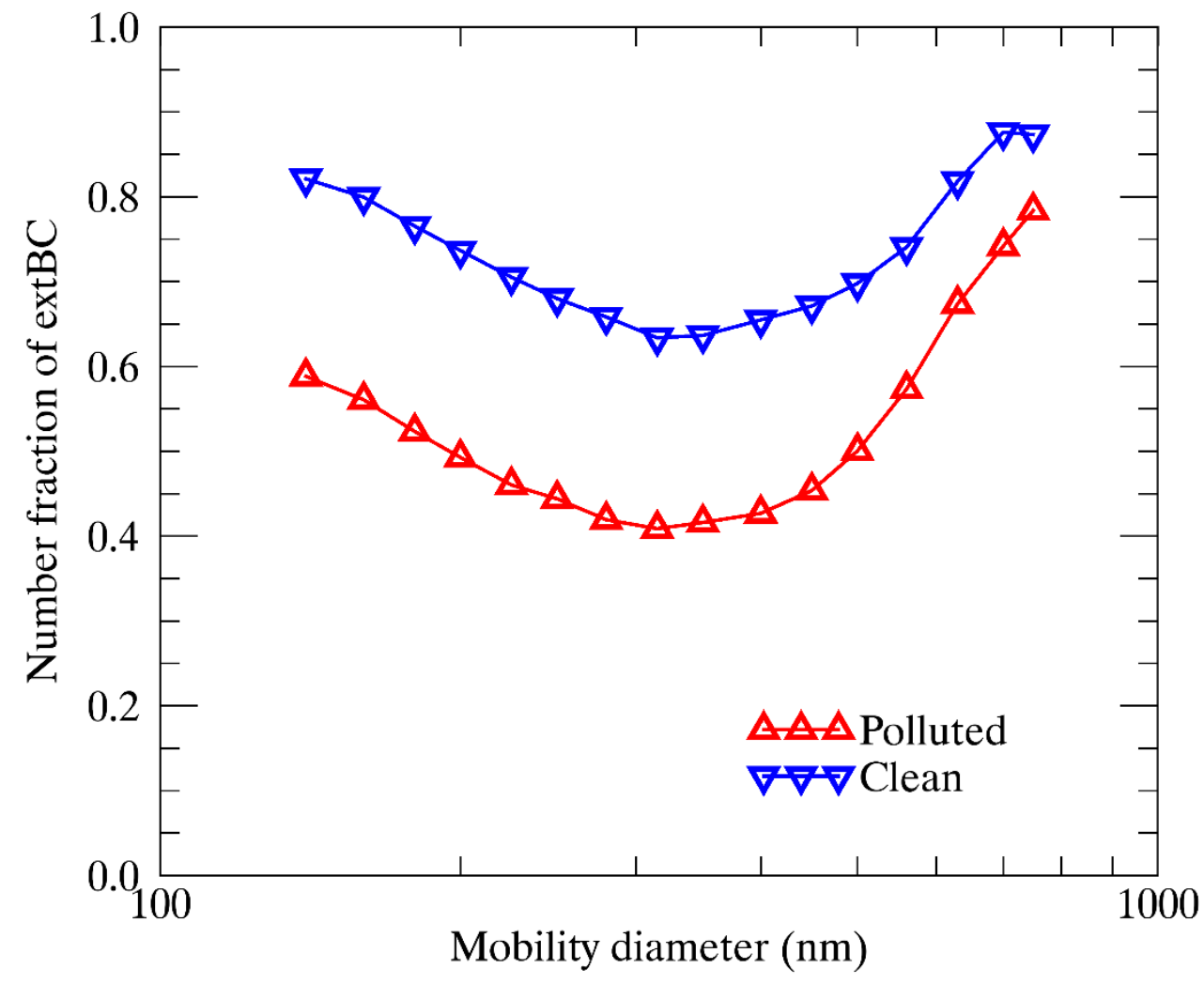

105 Fig. S9: The number fraction of ext $\mathrm{BC}$ as a function of the mobility diameter $\left(d_{\mathrm{mob}}\right)$ in the polluted episode (red upward triangles) and clean period (blue downward triangles). The fraction of ext $\mathrm{BC}$ is roughly calculated as the ratio of the ext $\mathrm{BC}$ number concentration to the sum of ext $\mathrm{BC}$ and internally mixed $\mathrm{BC}$ (int $\mathrm{BC}$ ) particles at each $d_{\text {mob. The effect of }}$ multicharged particles is not eliminated. However, the multicharged particles should result in a similar effect on ext $\mathrm{BC}$ and int $\mathrm{BC}$ at a given mobility. Thus, there should be few effects on the number fraction of $\operatorname{ext} \mathrm{BC}$. A detailed analysis of 110 the mixing states of size-resolved BC particles will be performed in our future studies. 
Table S1: The typical mass-equivalent diameters $\left(d_{\mathrm{me}}\right)$ and corresponding masses of ext $\mathrm{BC}$ at different mobility sizes $\left(d_{\mathrm{mob}}\right)$ selected by the DMA in the whole campaign. Three delay time thresholds of $2.0,1.2$, and $0.4 \mu$ s are employed to discriminate ext $\mathrm{BC}$.

\begin{tabular}{cccccccc}
\hline$d_{\text {mob }}(\mathrm{nm})$ & \multicolumn{3}{c}{$d_{\mathrm{me}}(\mathrm{nm})$} & & \multicolumn{3}{c}{ mass $(\mathrm{fg})$} \\
\cline { 2 - 3 } \cline { 6 - 7 } \cline { 6 - 7 } & $t_{\mathrm{lag}}<2.0 \mu \mathrm{s}$ & $t_{\mathrm{lag}}<1.2 \mu \mathrm{s}$ & $t_{\mathrm{lag}}<0.4 \mu \mathrm{s}$ & & $t_{\mathrm{lag}}<2.0 \mu \mathrm{s}$ & $t_{\text {lag }}<1.2 \mu \mathrm{s}$ & $t_{\mathrm{lag}}<0.4 \mu \mathrm{s}$ \\
\hline 140 & 88.8 & 88.4 & 86.7 & & 0.66 & 0.65 & 0.61 \\
160 & 97.5 & 97.6 & 96.2 & & 0.87 & 0.88 & 0.84 \\
180 & 106.2 & 106.6 & 105.0 & & 1.13 & 1.14 & 1.09 \\
200 & 115.6 & 116.2 & 114.2 & & 1.46 & 1.48 & 1.40 \\
225 & 127.9 & 128.8 & 125.8 & & 1.97 & 2.01 & 1.88 \\
250 & 140.5 & 141.6 & 138.1 & & 2.62 & 2.67 & 2.48 \\
280 & 155.8 & 156.9 & 152.9 & & 3.56 & 3.64 & 3.37 \\
315 & 172.6 & 173.6 & 169.4 & & 4.85 & 4.93 & 4.58 \\
350 & 188.2 & 189.2 & 184.7 & & 6.28 & 6.38 & 5.94 \\
400 & 207.4 & 208.6 & 204.8 & & 8.41 & 8.55 & 8.10 \\
450 & 226.4 & 228.0 & 224.1 & & 10.94 & 11.17 & 10.61 \\
500 & 243.8 & 245.4 & 241.2 & & 13.65 & 13.92 & 13.23 \\
560 & 262.6 & 265.4 & 262.0 & & 17.06 & 17.62 & 16.96 \\
630 & 283.2 & 286.1 & 285.3 & & 21.42 & 22.07 & 21.88 \\
700 & 305.1 & 307.9 & 309.8 & & 26.76 & 27.52 & 28.03 \\
750 & 319.6 & 322.3 & 323.4 & & 30.76 & 31.57 & 31.87 \\
\hline
\end{tabular}

